Messrs. John Wheldon AND Co., 38 Great Queen Street, Kingsway, W.C., have just issued a catalogue of important books and papers on cryptogamic botany they are offering for sale. The works are arranged conveniently under three main divisions-economic, geographical, and general-each of which is subdivided to facilitate search for works on any particular subjects embraced by the catalogue.

\section{OUR ASTRONOMICAL COLUMN.}

Comet. igi6a (Neujmin).-Copenhagen Postcards Nos. I 3 and I4 give orbits and ephemerides for this comet calculated by M. J. Fischer-Petersen and Mile. J. M. Vinter-Hansen. The earlier orbit is based on observations made at Yerkes (February 29), Greenwich (March I), and at Bamberg on March 3. The second, given below, depends on the Yerkes and Bamberg positions, and observations made at Bergedorf on March 5 :-

Perihelion Passage (T), I916, March; 9.4I7 G.M.T.

$$
\left.\begin{array}{rlrl}
\omega & =191^{\circ} & 9^{\prime} & 87^{\prime \prime} \\
\delta & =325^{\circ} & 24^{\prime} & 10^{\prime \prime} \\
i & =16^{\circ} & \mathbf{I}^{\prime} & 48^{\prime \prime}
\end{array}\right\} \text { I916.0 }
$$$$
\text { R.A. Dec. }
$$$$
\text { b. m. s. }
$$

March 15

$$
954 \mathrm{I}+55^{\circ} \cdot 2
$$$$
\text { March } 21
$$
17 $\begin{array}{llll}7 & 17 & 5 & 1 \cdot 2\end{array}$

9 IO $56+3$ III. $\begin{array}{lllll}12 & 57 & 2 & 1 & 1\end{array}$ 156 I 26.7

The orbit is apparently periodic in short period. The comet is fainter than II.o mag.

COMET I9I5e (TAYLOR).-A new orbit and ephemeris for this comet has been calculated by M. J. Braae from observations made at Rome, December 5, I915, at Arcetri and Copenhagen, January 11,1916 , and at Bamberg and Copenhagen on February 20. The new orbit only differs slightly from the earlier elliptical orbit (NaTuRe, January 2u) :-

Perihelion Passage $(T)=1916$, January 30.9122 G.M.T.

$$
\begin{aligned}
& \text { Epoch } 1916 \mathrm{Jan} .0^{\circ} 5 \text { (i. M.T. } \\
& M_{0}=355^{\circ} 17^{\prime} 34^{\circ} 6^{\prime \prime} \\
& \mu=557^{\prime \prime} 191 \\
& \log \alpha=0536002 \\
& \mathrm{U}=2325^{\circ} 95 \text { days }
\end{aligned}
$$

$(6 \cdot 37$ years $)$.

$$
\begin{aligned}
& \text { Equinox 1916.0 } \\
& \omega=354^{\circ} 47^{\prime} 54^{\circ} 9^{\prime \prime} \\
& \delta 6=11353 \quad 57^{\circ} 6 \\
& i=153 \mathrm{I} 51^{\circ} \mathrm{O} \\
& \phi=33733^{\circ} 9
\end{aligned}
$$

The comet is very weak, having been about 12 mag. on February 20.

From Bergedorf, Prof. Schorr has reported (Circular No. 503, Astronomische Nachrichten) that the nucleus of this comet. has divided into two portions. The nuclei were of unequal brightness, about magnitudes I I and 13. Their positions were:-Distance, I4"; position angle, $169^{\circ}$ and $17^{\prime \prime}$ and $25^{\circ}$, on February 19 and 29 respectively. On the latter date the following nucleus was the weaker.

According to a note in the current number of the Observatory, Prof. E. E. Barnard observed the double nucleus on February 9, the separation being $10^{n}$.

Variable Stars in the Vicinity of $\mathrm{R}$ Corona Australis.-This region is under careful scrutiny, not only at Helwân, but also at the Union Observatory, Johannesburg. In Circular No. 3I both R Coronæ and the nebula are stated to be variable over a wide range. The observations of these objects are to be discussed later. Thirty-three new variable stars have been detected in the region.

A Possible Deflection of Light by a Moving Medium.-Prof. P. Zeeman has published $(K$.

Akademie van Wetenschapen, vol. xviii., pp. 71I-5) an investigation of the propagation of light-waves along a velocity gradient in a moving medium specially in relation to solar phenomena. From a consideration of the Lorentz dispersion term in the Fresnel coefficient, it is demonstrated that the simultaneous existence of velocity gradients and anomalous dispersion in gases that are extremely rare (e.g. the absorbing vapours giving rise to the finest lines in the solar spectrum), and without density gradients, may give rise to a deflection of light.

\title{
A TUNGSTEN TARGET FOR X-RAY TUBES.1
}

CREAT advances have recently been made in the production of X-rays, chiefly by the employment of very heavy currents. The exposures necessary for producing radiographs of the thorax have been reduced from minutes to fractions of a second.

To make this possible, much attention has been devoted to the target or anti-kathode, which is the critical part of the tube, for here it is that the focus of the kathode stream strikes, and the energy of the bombarding electrons is transformed into $\mathrm{X}$-radiation.

The early English tubes were furnished with substantial targets of platinum, but in the later foreign tubes with which the market was flooded the platinum was often reduced to a sheet of very thin foil laid upon a plate of nickel. For weak currents, and with an imperfectly focused kathode stream, this plan answered moderately well, but if heavy currents were used the heat generated at the focus was often so great that the platinum skin alloyed with the nickel

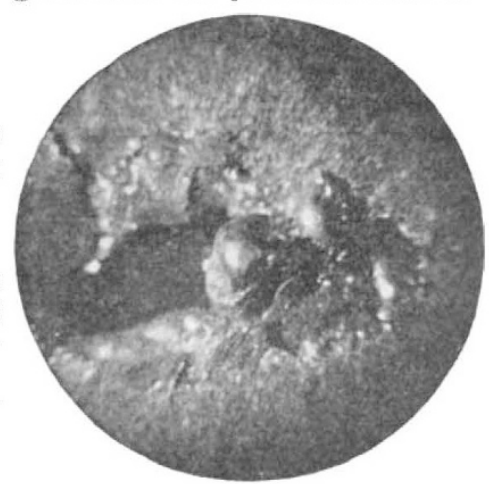

Platinised nickel target danaged by the kathode focus.

perties of pure metallic tungste perties in tungsten, now produced in quantity for the manufacture of metal filament lamps, and its suitability for the purpose was at once recognised, the metal having a fusing point of about $3000^{\circ} \mathrm{C}$, , as against $1750^{\circ} \mathrm{C}$. for platinum. Tungsten is also very tough, and does not readily disintegrate by the kathodic discharge (kathode sputtering); its atomic weight, 180 , is not much below that of platinum.

The British Thomson-Houston Company, Ltd., has introduced a special target of this metal that is being largely used by manufacturers of X-ray tubes. The tungsten is in the form of a thick button brazed into a solid block of copper, in some cases weighing as much as half a pound; this forms a lasting and efficient target, even when heavy currents are used

1 "Quantitative Measurements of the Conversion of Kathode Rays into Journal of the Röntgen Society, No. 24, vol. vi.

NO. 242O, VOL. 97] 